\title{
A comparison of the programme coverage of two therapeutic feeding interventions implemented in neighbouring districts of Malawi
}

\author{
Kate Sadler $1,2, *$, Mark Myatt ${ }^{3}$, Teshome Feleke ${ }^{1}$ and Steve Collins ${ }^{1,2}$ \\ 'Valid International Ltd, Unit 14, Standingford House, 26 Cave Street, Oxford OX4 3TN, UK: \\ ${ }^{2}$ Centre for International Child Health, Institute of Child Health, London WCIN 1EH, UK: ${ }^{3}$ Division of \\ Epidemiology, Institute of Ophthalmology, University College London, London ECIV 9EL, UK
}

Submitted 16 March 2006: Accepted 14 November 2006: First published online 30 April 2007

\begin{abstract}
Objective: To compare therapeutic feeding programme coverage for severely malnourished children achieved by a community-based therapeutic care (CTC) programme and a therapeutic feeding centre (TFC) programme operating in neighbouring districts in Malawi.

Design: Two surveys were implemented simultaneously one in each of the two programme areas. Each survey used a stratified design with strata defined using the centric systematic area sample method. Thirty $100 \mathrm{~km}^{2}$ quadrats were sampled. The community or communities located closest to the centre of each quadrat were sampled using a case-finding approach. Cases were defined as children aged under 5 years with $\leq 70 \%$ of the weight-for-height median or bilateral pitting oedema. Receipt of treatment was ascertained by the child's presence in a therapeutic feeding programme or by documentary evidence. Coverage in each quadrat was estimated in two ways, a period estimate that provides an estimation of coverage for the recent period preceding the survey and a point estimate that provides an estimation of coverage at the exact point in time of the survey.

Results: Overall the period coverage was $24.55 \%$ (95\% confidence interval $(\mathrm{CI})=17.8-31.4 \%)$ in the TFC programme and $73.64 \%(95 \% \mathrm{CI}=66.0-81.3 \%)$ in the CTC programme. The point coverage was $20.04 \%(95 \% \mathrm{CI}=13.8-26.3 \%)$ in the TFC programme and $59.95 \%(95 \% \mathrm{CI}=51.4-68.5 \%)$ in the CTC programme.

Conclusions: In this context, CTC gave substantially higher programme coverage than a TFC programme. Given effective treatment, this enabled higher impact of CTC on severe malnutrition in this population.

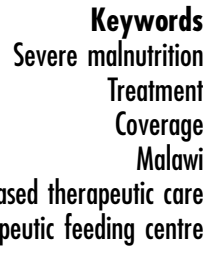

Until recently, therapeutic feeding centres (TFCs) have been the only accepted mode of intervention to address severe malnutrition during humanitarian interventions. These centres often provide high-quality individual patient care using highly appropriate diets and medical treatments based on the World Health Organization (WHO) protocol for the treatment of severe malnutrition ${ }^{1}$. However, centre-based treatment requires that the patient and the carer leave their home and travel to a centre, often many miles away, where they must stay for the duration of treatment. On average, treatment takes 27 days to recovery in well-run $\mathrm{TFCs}^{2}$ and longer in others ${ }^{3}$. These high opportunity costs limit programme coverage rates and therefore limit the population-level impact of centre-based programmes ${ }^{4,5}$. Since the 1970 s, these problems have prompted many to try, with varying success, to move the locus of treatment away from hospitals towards the community, either into simpler nutrition rehabilitation centres, existing primary health care clinics or into the homes of those affected ${ }^{6-8}$.

In 2001, we proposed a new intervention model called Community-based Therapeutic Care (CTC) ${ }^{9}$. CTC programmes treat the vast majority of severely malnourished people in their homes and aim to restrict in-patient care to only those suffering from acute malnutrition with complications ${ }^{10}$. CTC programmes to date have produced positive clinical outcomes ${ }^{11,12}$ with mortality rates in the first 7000 severely malnourished patients treated of $<5 \%$, considerably better then international standards for the treatment of severe acute malnutrition ${ }^{3}$. They use decentralised networks of out-patient treatment sites to provide a take-home food ration known as ready-to-use therapeutic food ${ }^{13}$ as well as routine medicines. Large numbers of community nutrition volunteers supported by outreach workers find and refer cases to programme sites and follow up patients at home. By providing easy access 
and limiting the opportunity costs associated with enrolment in the programme, the CTC model aims to increase the coverage and therefore the impact of humanitarian therapeutic feeding interventions.

Coverage is a vital determinant of impact in any humanitarian intervention. The Sphere Project was launched in 1997 to develop a set of universal minimum standards in core areas of humanitarian assistance. The latest revision of the Sphere Project incorporates coverage standards of 50\% for rural populations, $75 \%$ for urban populations and $90 \%$ for camp populations as a key indicator of programme performance $^{3}$. Until recently, coverage was estimated using an adaptation of the coverage survey method used in the WHO's Expanded Programme on Immunisation ${ }^{14,15}$. This method uses a two-stage cluster sampling approach and a sampling procedure, called probability proportional to size (PPS). Sample size restrictions imposed by the fact that severe malnutrition is a relatively rare condition means that such survey estimates tend to lack useful precision. In addition, PPS sampling locates the bulk of data collection in the most populous communities. This may leave areas of low population density unsampled, i.e. those areas consisting of communities likely to be distant from health facilities, feeding centres and distribution points. This may cause surveys to evaluate coverage as being adequate even when coverage is poor or non-existent in areas outside urban centres $^{16}$. As part of the CTC programme, we have developed a new method of assessing coverage ${ }^{17}$. This method is simple and rapid to implement, provides a useful level of precision and allows examination of the geographical distribution of coverage. This study uses this method to compare the coverage achieved by a centrebased therapeutic feeding programme and a CTC programme operating in neighbouring districts in Malawi during 2003.

\section{Methods}

\section{Trial setting}

In February 2002, the Malawi government declared a national nutritional emergency and the United Nations
(UN) launched an international appeal for emergency assistance. The national Ministry of Health $(\mathrm{MoH})$ and the humanitarian community began to develop strategies for the treatment of the large numbers of severely malnourished that were predicted. Nationally, a strategy of upgrading 92 existing nutritional rehabilitation units (NRUs) across the country was adopted, with the aim of each NRU being able to provide centre-based therapeutic treatment according to $\mathrm{WHO}$ protocols ${ }^{1}$. The United Nations Children's Fund and several non-governmental organisations (NGOs) provided therapeutic products, training and support for this strategy. At the same time, an Irish NGO, Concern Worldwide, with the support of Valid International, was given permission by the MoH to pilot CTC in two of the 25 districts.

In March 2003, 7 months into implementation of both the national TFC strategy and the CTC programme in Dowa District, we implemented a study to compare the coverage of the CTC programme in Dowa with that of a centre-based therapeutic feeding programme for the treatment of severe malnutrition in the similar neighbouring district of Mchinji. Both districts are located in the central region of Malawi and had been subject to prolonged food shortages ${ }^{18}$.

Dowa and Mchinji Districts are similar in their demographic and socio-economic profiles (see Table 1) $19-22$

In accordance with the national strategy for the treatment of acute malnutrition, an international NGO provided training and support in Mchinji for two NRUs, based at government health facilities, to provide in-patient TFC treatment for children with severe malnutrition. An additional NRU, at a mission hospital run by the Christian Health Association of Malawi, was also given some support. All supported NRUs provided phase 1, 2 and 3 inpatient care for severely malnourished children according to WHO and national protocols ${ }^{23}$. In addition, a supplementary feeding programme was supported at each treatment site. The Mchinji programme typified the level of support being provided under the national strategy for the treatment of acute malnutrition.

Table 1 Demographic and socio-economic characteristics of the two study areas

\begin{tabular}{lll}
\hline & Dowa & Mchinji \\
\hline Size $\left(\mathrm{km}^{2}\right)$ & 3041 & 3156 \\
Population & 411000 & 324941 \\
Under-5 population & 73980 & 58489 \\
Main religion & $>90 \%$ Christian & $>90 \%$ Christian \\
Percentage population in formal employment & $1 \%$ & $8 \%$ \\
Percentage land under cultivation & $47 \%$ & $62 \%$ \\
Prevalence of global acute malnutrition (weight-for-height Z-scores) & $4.5 \%$ (February 2003) & $2.9 \%$ (December 2002) \\
Average population per health centre & 20360 & 26839 \\
Average population per doctor & 101000 & 348903 \\
Leading cause of mortality & Malaria & Malaria \\
Hospital beds per 1000 population & 0.9 & 1.1 \\
General ration distribution start date & June 2002 & June 2002 \\
General ration distribution target population (no. of households) & 20218 & 10232 \\
\hline
\end{tabular}


In Dowa, the CTC intervention established four small stabilisation centres for the treatment of severe malnutrition with complications within mission- and government-run hospitals and NRUs. These provided phase 1 in-patient care according to WHO and national protocols. In addition, 18 out-patient treatment sites were established within health centres across the district. Each out-patient treatment site implemented an out-patient therapeutic programme ${ }^{9}$ and a supplementary feeding programme NGO staff made up mobile support teams which rotated around treatment points and supported the $\mathrm{MoH}$ staff with service delivery.

Programmes in both districts were established within existing $\mathrm{MoH}$ structures and therapeutic care was delivered by $\mathrm{MoH}$ staff in health centres and NRUs. Table 2 describes the additional inputs provided by each NGO and UN organisations to support the MoH staff during programme implementation.

\section{Survey design}

Two surveys were implemented simultaneously one in each of Dowa and Mchinji Districts. Each survey used a stratified design with strata defined using the centric systematic area sample method according to the methods we have described previously ${ }^{17}$. This method involves dividing the survey area into non-overlapping squares of equal area (quadrats) and sampling the community or communities located closest to the centre of each quadrat. A 1:50 000 scale map of each district was available from the 1998 Malawi national census. A $10 \mathrm{~km} \times 10 \mathrm{~km}$ grid was overlaid onto each map. All quadrats with more than half of their area inside the districts were sampled. In each district, thirty $100 \mathrm{~km}^{2}$ quadrats were sampled. The selected quadrats covered $3000 \mathrm{~km}^{2}$. Of the total district land areas, 4.9 and 1.3\% fell outside the surveyed areas in Mchinji and Dowa, respectively. Communities located closest to the centre of each quadrat were sampled using a case-finding approach. The number of communities sampled from each quadrat was dictated by the number of communities in that quadrat that could be sampled by a survey team in a single day. This varied between quadrats and depended on the size of each community (in terms of both population and physical extent) and the distances between communities. Once sampling started in a community, it continued until no further cases could be found. No communities were partially sampled.

\section{Case finding}

For the within-community samples, four methods of casefinding severely malnourished children were tested ${ }^{17}$. The method adopted involved going to homes and screening all children whom the community health worker

Table 2 Programme inputs provided by supporting agency in the two study areas

\begin{tabular}{|c|c|c|}
\hline & CTC programme Dowa & TFC programme Mchinji \\
\hline NGO staff: direct patient care & $\begin{array}{l}1 \times \text { nutritionist (expatriate) } \\
\text { Health centre/community support: } \\
2 \times \text { team leaders } \\
2 \times \text { nurses } \\
2 \times \text { registration staff } \\
2 \times \text { community educators } \\
2 \times \text { drivers } \\
\text { NRU support: } \\
2 \times \text { nurses } \\
2 \times \text { feeding attendants }\end{array}$ & $\begin{array}{l}1 \times \text { doctor/nutritionist (expatriate) } \\
1 \times \text { nurse } \\
1 \times \text { nutritionist } \\
1 \times \text { driver }\end{array}$ \\
\hline $\begin{array}{l}\text { Consultant staff: CTC development } \\
\text { and monitoring }{ }^{*}\end{array}$ & $\begin{array}{l}1 \times \text { doctor (expatriate) } \\
1 \times \text { nutritionist (expatriate) } \\
1 \times \text { anthropologist (expatriate) }\end{array}$ & \\
\hline Food & $\begin{array}{l}\text { To all health centres and NRUs: } \\
\text { RUTFs } \\
\text { Corn-soya blend (from WFP) } \\
\text { To all NRUs: } \\
\text { Therapeutic milks (from UNICEF) }\end{array}$ & $\begin{array}{l}\text { To all NRUs: } \\
\text { Therapeutic milks (from UNICEF) } \\
\text { Corn-soya blend (from WFP) }\end{array}$ \\
\hline Medicines & $\begin{array}{l}\text { IDA drug kits that include the routine } \\
\text { medicines required to treat severe } \\
\text { malnutrition provided to each } \\
\text { treatment site (a proportion } \\
\text { from UNICEF) }\end{array}$ & $\begin{array}{l}\text { IDA drug kits that include the routine } \\
\text { medicines required to treat severe } \\
\text { malnutrition provided to each } \\
\text { treatment site (from UNICEF) }\end{array}$ \\
\hline Non-food items & Soap & \\
\hline Transport & $2 \times$ four-wheel drive vehicle & $1 \times$ four-wheel drive vehicle \\
\hline $\begin{array}{l}\text { Method of motivation for partner } \\
\text { MoH staff in helth centres, } \\
\text { NRUs and community }\end{array}$ & $\begin{array}{l}\text { Training and ongoing support from } \\
\text { programme staff during programme } \\
\text { implementation }\end{array}$ & $\begin{array}{l}\text { Training and ongoing support } \\
\text { from programme staff during } \\
\text { programme implementation }\end{array}$ \\
\hline
\end{tabular}

CTC - Community-based Therapeutic Care; TFC - Therapeutic Feeding Centre; NGO - non-governmental organisation; MOH Ministry of Health; NRU - Nutritional rehabilitation unit; RUTF - ready-to-use therapeutic food; WFP - World Food Programme; UNICEF - United Nations Children's Fund; IDA - International Dispensary Association.

${ }^{*}$ Consultant staff were research staff on short-term support visits. 
identified as thin, sick or oedematous. Additional children were also identified by mothers in each of the screened households. Where community health workers or mothers indicated that a child was absent due to admission to an NRU or attendance at a therapeutic feeding programme, the survey team followed-up the child at the programme site or returned to the child's home the following day.

\section{Case definitions}

Cases were defined as children aged under 5 years with a weight-for-height $\leq 70 \%$ of the National Center for Health Statistics (NCHS) reference population median weight-forheight $^{24}$ or bilateral pitting oedema. Receipt of treatment was ascertained by the child's presence in a therapeutic feeding programme or by documentary evidence (i.e. possession of a programme card or identity bracelet).

The survey used two different case definitions. The first case definition was used to provide an estimation of coverage for the recent period preceding the survey (period estimate). This case definition is equivalent to that traditionally used by agencies such as WHO and Medécins Sans Frontières ${ }^{14,15}$ to estimate coverage in centre-based programmes and to that used in coverage standards laid out by the Sphere Project ${ }^{3}$. It included all those cases that were malnourished at the time of the survey and, in addition, it included all children registered in a therapeutic feeding programme. This definition therefore included children registered in the programme who were no longer severely malnourished but had not yet attained the treatment programme discharge criteria. In this case definition, cases were defined as children aged under 5 years with a weight-for-height $\leq 70 \%$ of the NCHS reference population median weight-for-height ${ }^{24}$ or bilateral pitting oedema plus any children registered in a therapeutic feeding programme.

The second case definition was used to provide an estimation of coverage at the exact point in time of the survey (point estimate). This case definition included only cases that were malnourished at the time of the survey. In this case definition, cases were defined as children aged under 5 years with a weight-for-height $\leq 70 \%$ of the NCHS reference population median weight-for-height ${ }^{24}$ or bilateral pitting oedema.

\section{Program coverage}

Coverage in each quadrat was estimated in two ways, a period estimate that used the first case definition and a point estimate that used the second case definition. For each estimate, coverage was calculated as the ratio of cases receiving treatment found in the sample to the total number of cases found in the sample (see Fig. 1). Overall coverage was estimated by treating each quadrat as a stratum in a stratified sample ${ }^{25}$ with sample weights derived from the population of the communities sampled in each quadrat.

\section{Data bandling}

Data were entered, checked and cleaned using EpiInfo $\mathrm{v} 6.04 \mathrm{~d}^{26}$, and analysed using the R Language for Data Analysis and Graphics ${ }^{27}$. The spatial distribution of coverage was investigated by estimating coverage in each quadrat and plotting the data using histograms and mesh maps.

\section{Results}

\section{Period estimation of coverage}

Overall the period coverage was 24.55\% (95\% confidence interval $(\mathrm{CI})=17.8-31.4 \%)$ in Mchinji and $73.64 \%(95 \%$ $\mathrm{CI}=66.0-81.3 \%)$ in Dowa. The distribution of perquadrat coverage for each district is shown in Figs 2 and 3. Coverage ranged between zero (in five quadrats) and 50\% (in two quadrats) in Mchinji and between zero (in one quadrat) and 100\% (in 10 quadrats) in Dowa.

\section{Point estimation of coverage}

Overall the point coverage was 20.04\% (95\% CI $=13.8-$ 26.3\%) in Mchinji and 59.95\% (95\% CI = 51.4-68.5\%) in Dowa. Coverage ranged between zero (in nine quadrats) and 50\% (in one quadrat) in Mchinji and between zero (in four quadrats) and 100\% (in 10 quadrats) in Dowa.

\section{Patterns of coverage}

The spatial distribution of per-quadrat period coverage for each district is shown in Figs 4 and 5 . Full grey squares represent $100 \%$ coverage, whilst an empty/white square represents $0 \%$ coverage. The approximate locations of the

1. Period coverage is calculated using the following formula:

number attending the feeding programme
(number of cases not attending the feeding programme
+ number attending the feeding programme)

2. Point coverage is calculated using the following formula:

$\frac{\text { number of cases attending the feeding programme }}{\text { total number of cases }} \times 100$

Fig. 1 Formulae for calculating coverage 
Comparison of the coverage of two therapeutic feeding interventions

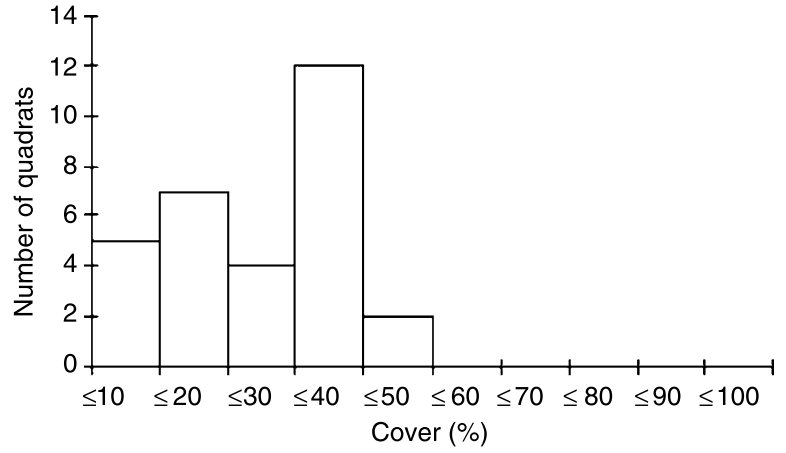

Fig. 2 Distribution of per-quadrat period coverage in the TFC (therapeutic feeding centre) programme in Mchinji

NRUs in each district are marked, and thin black lines indicate the approximate location of major roads. The spatial distribution of per-quadrat point coverage for each district is very similar to that shown for the period coverage in Figs 4 and 5 and therefore is not presented here. Both period and point coverage appeared more uniform in Dowa than in Mchinji. In Mchinji, five quadrats (13\%) had zero period coverage and only two quadrats (7\%) met the Sphere coverage standard ${ }^{3}$. In Dowa, period coverage met the Sphere Project standard in 27 out of 30 (90\%) quadrats, and in only one quadrat (3\%) was it zero. In Mchinji, nine quadrats $(30 \%)$ had zero point coverage and in only one quadrat $(3 \%)$ was coverage $>50 \%$. In Dowa, point coverage was $>50 \%$ in 21 out of $30(70 \%)$ quadrats, and in only four quadrats (13\%) was it zero.

\section{Discussion}

Historically, evaluation of selective feeding programmes has focused on cure and mortality rates ${ }^{28}$, and the coverage of therapeutic programmes has either gone unmeasured $^{2}$ or, on the few occasions where it has been assessed, has been extremely low ${ }^{5}$. Figure 6 demonstrates the importance of coverage as an indicator of impact. High coverage but low cure rate programmes can meet a higher proportion of need in a population than low coverage but high cure rate programmes. In order to maximise impact,

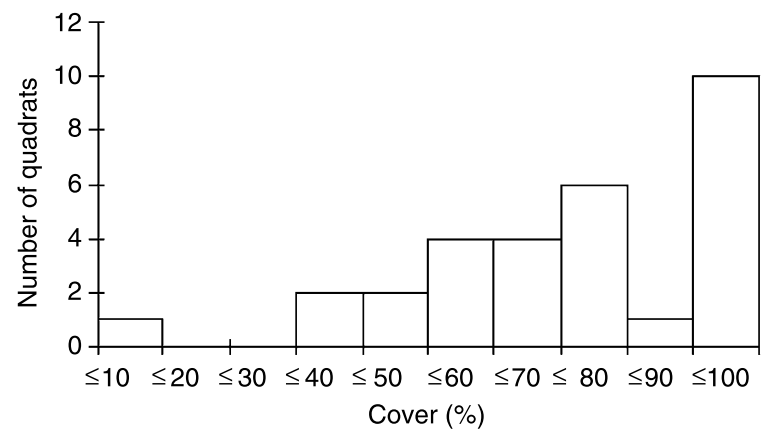

Fig. 3 Distribution of per-quadrat period coverage in the CTC (community-based therapeutic care) programme in Dowa

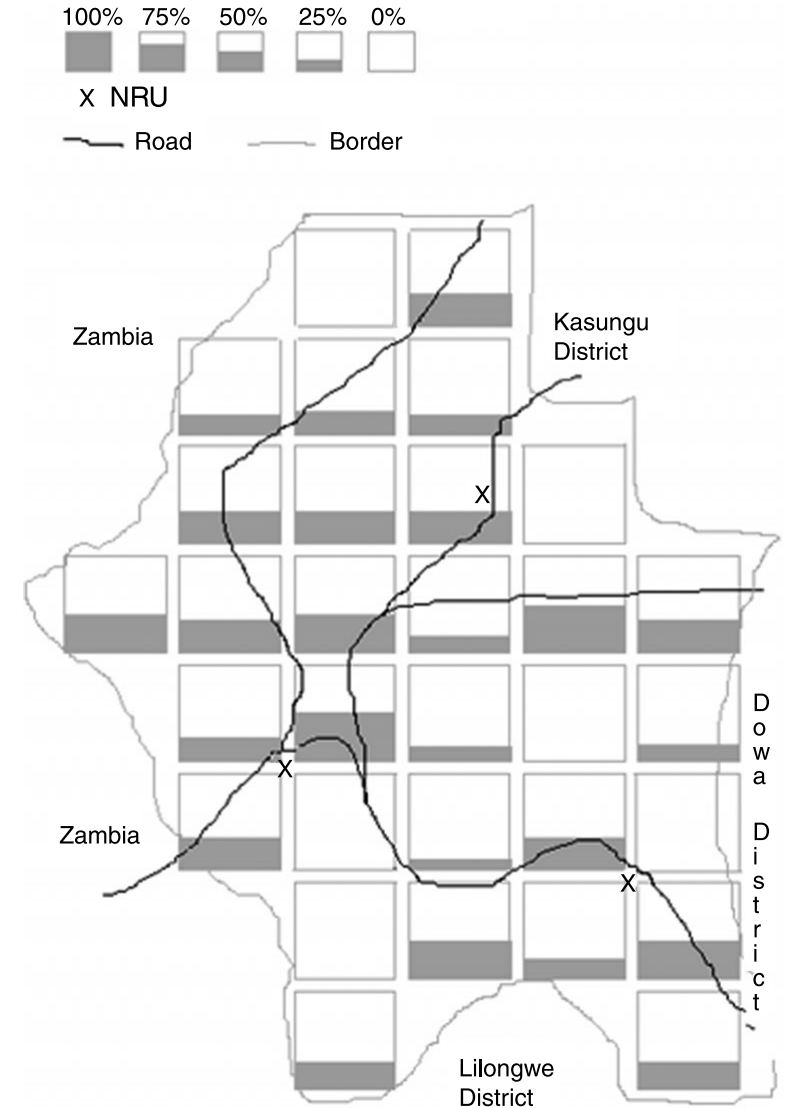

Fig. 4 Spatial distribution of per-quadrat period coverage in the TFC (therapeutic feeding centre) programme in Mchinji (NRU nutritional rehabilitation unit)

programmes must have both high coverage and high cure rates. The importance of coverage has recently been acknowledged with the addition of coverage indicators in the second edition of Sphere ${ }^{3}$.

In this study, both the period and point coverage estimations were substantially higher for the CTC programme in Dowa than for the TFC programme in Mchinji. For the CTC programme, both point coverage and period coverage, the measure that is comparable with existing international measures of coverage, exceeded the Sphere standard of $>50 \%$ for rural areas. For the TFC programme, neither coverage estimation met this standard. In March 2003, the CTC programme had achieved cure rates of $70 \%{ }^{29}$. In combination with a period coverage of $74 \%$, this indicates that the programme met 52\% (95\% $\mathrm{CI}=46-57 \%)$ of the need of the severely malnourished population in Dowa. Cure rates for the TFC programme in Mchinji were not available for this paper, but even if the cure rate was $100 \%$ this programme would have met only $25 \%(95 \% \mathrm{CI}=18-31 \%)$ of need.

CTC programme coverage also appeared to be more evenly spread across the district than in the TFC programme in Mchinji. Here, TFC programme coverage reflected access to treatment points, being highest around the NRUs and following the main routes of 


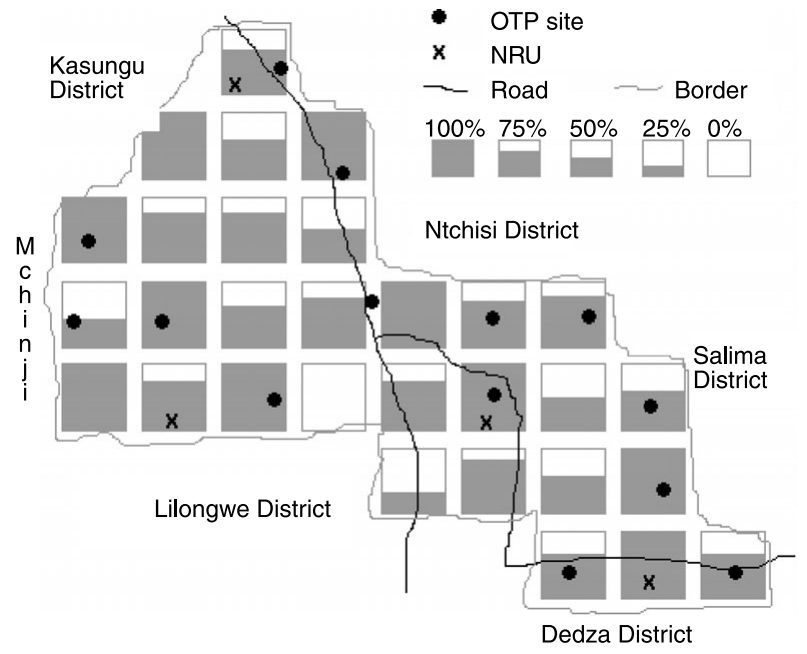

Fig. 5 Spatial distribution of per-quadrat period coverage in the CTC (community-based therapeutic care) programme in Dowa. (OTP - out-patient therapeutic programme; NRU - nutritional rehabilitation unit)

communication. This suggests that the decentralised CTC out-patient treatment model decreased barriers to access by reducing the distances people had to travel to attend treatment centres and reducing the need for carers to stay away from home.

It is important to remember that this was not a controlled study and that the CTC programme in Dowa received substantially higher levels of support and inputs than the TFC programme in Mchinji (see Table 2). Preliminary data indicate that the monetary cost per child treated in this CTC programme during the emergency set up period was US $\$ 197^{30}$. We do not have equivalent cost data from the TFC programme in Mchinji for comparison and there are few data available generally on the cost of TFC interventions. The little information that is available indicates that TFC care can cost anything between US\$156 and US $\$ 355$ per child treated ${ }^{31,32}$.

Relative costs and inputs, however, may not be the most important factor in achieving high coverage rates. It seems

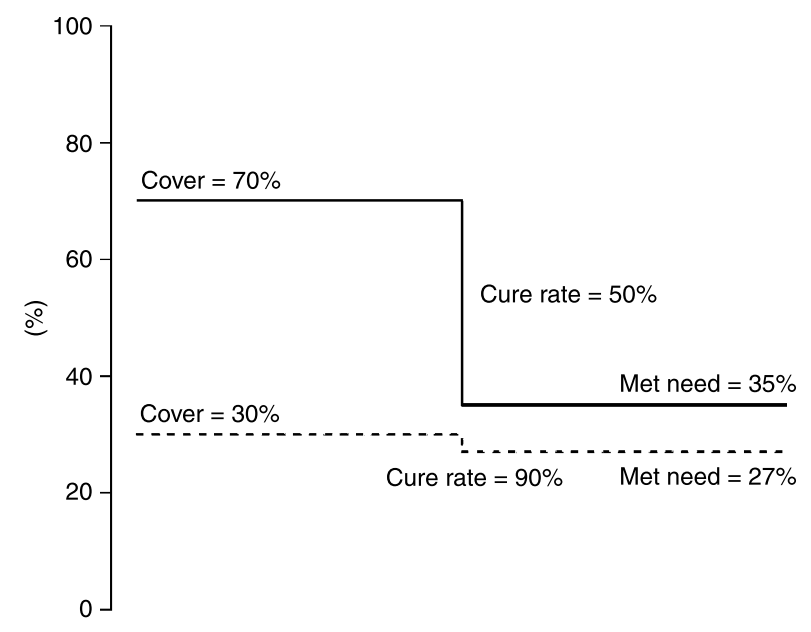

Fig. 6 Coverage, cure rate and impact that the decentralised programme design and the low opportunity costs associated with accessing treatment were very important influences on CTC programme uptake and coverage. Sociological studies of programme recipients in both Mchinji and Dowa indicated a marked preference for the CTC out-patient treatment model. Recipients highly valued the minimal disruption to normal lives associated with CTC treatment. This allowed them to continue farming, looking after other children and attending to daily household chores. In contrast, interviews with TFC recipients highlighted worries over prolonged separation from farms, other children and spouses. Prolonged separation of husbands and wives increased marital disharmony and jealousy, and was responsible for increasing default rates ${ }^{33}$. The persistence of high CTC coverage rates in 2004 (period coverage $72.2 \%$ ), when the CTC programme was progressively handed over to $\mathrm{MoH}$ control and external inputs substantially reduced ${ }^{34}$, also supports the view that the high coverage rates in the CTC programme were due to improved access to services and reduced opportunity cost to carers more than to the level of inputs provided.

\section{Conclusion}

In this context, the CTC model of care was associated with substantially higher programme coverage than the TFC model. CTC met the Sphere coverage standard of $>50 \%$ for overall coverage as well as in the majority of quadrats. The TFC programme failed to meet this standard in all but two of the 30 quadrats. Cure rates in the CTC programmes were also high, and the combination of high coverage and high cure rates resulted in a substantially higher impact of the CTC programme than that demonstrated by the TFC programme. The results also suggest that the ease of access to treatment and the low opportunity cost associated with the CTC out-patient treatment are important factors for increasing programme coverage.

In order to evaluate whether this comparative advantage over TFC programmes exists in other settings, future CTC and TFC programmes should examine coverage and cost-effectiveness in operational rather than research contexts.

\section{Acknowledgements}

Sources offunding: This work was undertaken as part of the Community Therapeutic Care Programme, a collaboration between Valid International and Concern Worldwide. It was supported financially by the World Health Organization; the United States Agency for International Development (USAID) through the Food and Nutrition Technical Assistance (FANTA) project/Academy for Educational Development (AED); Ireland Aid; and Brixton Health. 
Conflict of interest declaration: There are no conflicts of interest declared.

Authorship responsibilities: K.S. and T.F. implemented the field work for this study. In addition K.S. has been the leading author for the write up and has led the revision of various drafts. M.M. designed the methodology for this study and, with T.F., performed the majority of study data analysis. S.C. oversaw both the data analysis and the write up of the study.

\section{References}

1 World Health Organization (WHO). Management of Severe Malnutrition: A Manual for Physicians and Other Senior Health Workers. Geneva: WHO, 1999.

2 Grellety Y. Management of Severe Malnutrition in Africa. Aberdeen: University of Aberdeen, 2000.

3 Sphere Project Team. The Sphere Project: Humanitarian Charter and Minimum Standards in Disaster Response, 2nd ed. Geneva: The Sphere Project, 2004.

4 Van Damme W. Medical Assistance to Self-settled Refugees. Guinea 1990-1996. Studies in Health Services and Policy No. 11. Antwerp: ITG Press, 1998.

5 Van Damme W, Boelaert M. Medical assistance to self-settled refugees. Guinea (letter). Lancet 2002; 359: 260-1.

6 Cook R. Is hospital the place for the treatment of malnourished children? Journal of Tropical Pediatrics and Environmental Child Health 1971; 17: 15-25.

7 Bengoa JM. Nutrition rehabilitation centres. Journal of Tropical Pediatrics 1967; 13: 169-76.

8 Khanum S, Ashworth A, Huttly SR. Controlled trial of three approaches to the treatment of severe malnutrition. Lancet 1994; 344: 1728-32.

9 Collins S. Changing the way we address severe malnutrition during famine. Lancet 2001; 358: 498-501.

10 Collins S, Yates R. The need to update the classification of acute malnutrition. Lancet 2003; 362: 249.

11 Collins S, Sadler K. The outpatient treatment of severe malnutrition during humanitarian relief programmes. Lancet 2002; 360: 1824-30.

12 Emergency Nutrition Network (ENN). Proceedings of the Community-based Therapeutic Care Conference, Dublin, 8-10 October, 2003. Oxford: ENN, 2004.

13 Briend A, Lacsala R, Prudhon C, Mounier B, Grellety Y, Golden MHN. Ready-to-use therapeutic food for treatment of marasmus. Lancet 1999; 353: 1767-8.

14 Henderson RH, Sundaresan T. Cluster sampling to assess immunisation coverage: a review of experiences with a simplified sampling method. Bulletin of the World Health Organization 1982; 60: 253-60.

15 Boelaert M, Davis A, Le Lin B, Michelet M, Ritmeijer K, Van Der Kam S, et al. Nutrition Guidelines. Paris: Medécins Sans Frontières, 1995.

16 Valadex JJ. Assessing Child Survival Programs in Developing Countries - Testing Lot Quality Assurance Sampling. Boston, MA: Harvard University Press, 1991.
17 Myatt M, Feleke T, Collins S, Sadler K. A field trial of a survey method for estimating the coverage of selective feeding programs. Bulletin of the World Health Organization 2005; 83: $20-6$.

18 Malawi National Vulnerability Assessment Committee, SADC FANR Vulnerability Assessment Committee. Malawi Emergency Food Security Assessment Report. Malawi: Lilongwe, 2003.

19 Concern Worldwide, Malawi Ministry of Health. Nutrition and Mortality Survey Report: Dowa District Malawi. Ireland: Concern World Wide, 2003.

20 Save the Children (UK), Malawi Ministry of Health. Nutrition Survey Report: Salima District and Mchinji District. London: SCF UK, 2003.

21 Dowa District Assembly. Dowa District Socio-Economic Profile. Government of Malawi: Lilongwe, 2003.

22 Mchinji District Assembly. Mchinji District Socio-Economic Profile. Government of Malawi: Lilongwe, 2002.

23 Ministry of Health and Population. Manual for the Management of Acute Severe Malnutrition. Government of Malawi: Lilongwe, 2003.

24 National Center for Health Statistics (NCHS). NCHS Growth Curves for Children Birth to 18 Years. Washington: US Department of Health, Education and Welfare, Government Printing Office, 1979.

25 Moser CA, Kalton G. Survey Methods in Social Investigation, 2nd ed. London: Heinemann Educational Books, 1979.

26 Centers for Disease Control and Prevention (CDC). EpiInfo version 6: A Word Processing, Database, and Statsitics Program for Public Health on IBM-compatible Microcomputers. Atlanta, GA: CDC, 1995.

27 Ihaka R, Gentleman RR. A language for data analysis and graphics. Journal of Computational and Graphical Statistics 1996; 5: 299-314.

28 Sphere Project Team. The Sphere Project: Humanitarian Charter and Minimum Standards in Disaster Response. Geneva: The Sphere Project, 1999.

29 Concern Worldwide. CTC Programme Monitoring Report, Dowa District Malawi. Ireland: Concern World Wide, Lilongwe, 2003.

30 World Health Organization (WHO). Report of an Informal Consultation on the Community-based Management of Severe Malnutrition. Geneva: WHO, 2006.

31 Ashworth A, Khanum S. Cost-effective treatment for severely malnourished children: what is the best approach? Health Policy and Planning 1997; 12: 115-21.

32 European Community Humanitarian Office (ECHO). The Unit Cost Approach of Humanitarian Activities (Draft 1). Brussels: ECHO, 2001.

33 Bandawe CR, Kabwazi N. Cultural and Social Factors Affecting the Implementation and Success of the Community-Based Therapeutic Care (CTC) Approach to Treating Severe Malnutrition in Dowa, Malawi. Oxford: Valid International, 2003.

34 Wegerdt J. Selective Nutrition Programme Coverage Survey Report: Dowa District, Malawi. Oxford: Valid International, 2004. 